\title{
Diastolic Filling Characteristics in the Stage 12 to 27 Chick Embryo Ventricle
}

\author{
NORMAN HU, DAVID M. CONNUCK, BRADLEY B. KELLER, AND EDWARD B. CLARK \\ The Cook Research Laboratory, Division of Pediatric Cardiology, Department of Pediatrics, University of \\ Rochester School of Medicine and Dentistry, Rochester, New York 14642
}

\begin{abstract}
Cardiac output is affected by the diastolic filling characteristics of the ventricle. We hypothesized that the relative contributions of passive and active filling change as the ventricle develops from a smooth-walled tube to a trabeculated four-chamber heart. In stage 12 to 27 white Leghorn chick embryos, we simultaneously measured ventricular pressure with a servo-null micropressure system and dorsal aortic and atrioventricular velocities with a $20-\mathrm{MHz}$ pulsed-Doppler velocity meter. The analog waveforms were sampled at $500 \mathrm{~Hz}$ and converted to digital format via an analog/digital board. We partitioned diastole into passive and active components. The passive phase began with the return of the pressure curve to baseline and extended to the onset of the a-wave. The active phase began with the upstroke of the atrial velocity curve and extended to the upstroke of the ventricular pressure curve at end-diastole. Data are presented as mean $\pm \operatorname{SEM}(n \geq$ 6 at each stage) and analyzed by analysis of variance and regression analysis. At similar cycle lengths ranging from 480 to $600 \mathrm{~ms}(p>0.05)$, end-diastolic pressure increased from $0.24 \pm 0.02 \mathrm{~mm} \mathrm{Hg}$ at stage 12 to $0.55 \pm 0.01 \mathrm{~mm}$ $\mathrm{Hg}$ at stage 27. Passive and active filling volumes were 92 $\left(0.0038 \pm 0.0005 \mathrm{~mm}^{3}\right)$ and $8 \%\left(0.0004 \pm 0.0002 \mathrm{~mm}^{3}\right)$, respectively, at stage 12 and changed to $24(0.23 \pm 0.08$ $\left.\mathrm{mm}^{3}\right)$ and $76 \%\left(0.62 \pm 0.08 \mathrm{~mm}^{3}\right)$, respectively, at stage 27 . The ratio of passive to active filling volume decreased from 7.89 to 0.35 . Thus, active ventricular filling became dominant as the trabeculae formed in the embryonic ventricle. These observations define the diastolic filling characteristics of the embryonic heart during primary cardiac morphogenesis. (Pediatr Res 29: 334-337, 1991)
\end{abstract}

During the period of early rapid embryo growth and cardiac morphogenesis, the embryonic heart develops from a smoothwalled cardiac loop into a septated trabecular heart (1). Throughout this period, the embryonic heart pumps blood to meet the metabolic demand of the rapidly growing embryo. The cardiac cycle of the embryonic heart, like the mature heart, has atrial and ventricular systole and diastole.

Previous developmental studies of ventricular diastolic filling are limited to fetal life. As the fetus matures, the ratio of passive to active component of the diastolic filling increases (2). As part of our long-term investigation into the functional characteristics of the embryonic heart, we defined the changes in diastolic filling characteristics in the chick embryo during early cardiac morphogenesis. We found that the relative proportion of the passive component decreased and the active component increased from

Received May 22, 1990; accepted November 19, 1990

Correspondence: Norman Hu, University of Rochester, Pediatric Cardiology Box 631, 601 Elmwood Ave., Rochester, NY 14642.

Supported by NIH Grant HL42151 and New York State Affiliate of the American Heart Association $87-025 \mathrm{G}$ stage 12 to 27 . These observations define the diastolic filling characteristics of the embryonic heart during primary cardiac morphogenesis.

\section{MATERIALS AND METHODS}

Fertile white Leghorn chicken eggs were incubated blunt end up in a forced-draft $38.5^{\circ} \mathrm{C}$ incubator. The study group included Hamburger-Hamilton stage 12 to stage $27, \mathrm{~d} 2$ to $\mathrm{d} 5$ of a $21-\mathrm{d}$ incubation (3). We measured simultaneously ventricular pressure with a servo-null micropressure system, (World Precision Instruments, Sarasota, FL) and dorsal aortic blood flow and atrioventricular blood velocity with $20-\mathrm{MHz}$ pulsed-Doppler velocity meters $(4,5)$. Ventricular pressure was obtained from a $10-\mu \mathrm{m}$ glass cannula inserted into the lumen of the ventricle. Dorsal aortic blood flow was measured with a $750-\mu \mathrm{m}$ piezoelectric crystal positioned over the dorsal aorta adjacent to the sinus venosus (Fig. 1). Dorsal aortic diameter was measured with a filar micrometer eyepiece calibrated with a $10-\mu \mathrm{m}$ scribed glass standard. Atrioventricular blood velocity was measured with a second crystal positioned at the apex of the heart and aimed at the atrioventricular orifice (Fig. 1). At these stages of development, the atrioventricular orifice is undivided and demarcated by the anterior and posterior endocardial cushions (6).

The analog waveforms were sampled at $500 \mathrm{~Hz}$ by a Computerscope data acquisition analog/digital board (RC Electronics, Inc., Santa Barbara, CA) and 20-MHz AST PC computer. The converter offered 12 bits at an input range of -2.5 to $2.5 \mathrm{~V}$ and a real time sampling rate of $70 \mathrm{kHz}$. A resolution of 12 bits or 4096 levels would have a signal resolution of $1.2207 \mathrm{mV}$, or a velocity of $0.1351 \mathrm{~mm} / \mathrm{s}$ at the Doppler. Data were stored in a 51/4-inch 20-megabyte Bernoulli disk cartridge (Iomega Corp., Roy, UT)

We partitioned diastole into two components (Fig. 2). The passive phase began with the return of the pressure curve to baseline and extended to the onset of the active phase of the atrial flow waveform. The active phase began with the upstroke of the atrial velocity curve and extended to the upstroke of the ventricular curve at end-diastole. Portions of the passive and active volumes overlapped each other at faster heart rates (Fig. 3 at $T_{1}$ ). The demarcation between the passive and active velocities was dependent on heart rate, but was most clear as heart rate slowed (Fig. 3 at $T_{3}$ and $T_{4}$ ). Embryos were studied at similar heart rates to avoid the effect of heart rate on diastolic filling.

Although there may be some inertial (passive) flow after the passive phase, the inertial force that carries blood forward during passive flow is very small compared with viscous force that retards the flow. The kinematic viscosity of blood is $3.8 \times 10^{-2}$ $\mathrm{cm}^{2} / \mathrm{s}$. The Reynolds number is 1.6 with the estimation of effective diameter of the atrioventricular orifice as $0.50 \mathrm{~mm}$ and the mean active atrioventricular velocity as $12.33 \mathrm{~mm} / \mathrm{s}$ at stage $27(5,7)$. Because inertial force is important at a Reynolds number near 2000 , there is little contribution of inertial blood 
flow in this system. Thus, passive flow effectively ends with the onset of active flow.

We performed a frequency analysis of the Doppler system. The Doppler audio output signal was passed through a custombuilt Angioscan frequency analyzer (Unigon Industries, Inc., Mt. Vernon, NY) that displayed the frequency-analyzed directional audio signal. The audio signal of a stage 27 chick embryo showed two components of the atrioventricular velocity that corresponded with the passive and active demarcations of the Doppler atrioventricular velocity. The passive component of the audio signal was $730 \mathrm{~Hz}$, which was approximately double the $380 \mathrm{~Hz}$ found in the passive demarcation of the phasic spatial mean Doppler shift. The active component of the audio signal was $3200 \mathrm{~Hz}$, which was also approximately double the $1390 \mathrm{~Hz}$ in the active demarcation of the Doppler shift. Inasmuch as both active and passive velocities have a parabolic flow velocity profile, the acoustic beam of the Doppler system covered the entire crosssection of the atrioventricle and did not incorporate any extraneous flow.

Dorsal aortic blood flow, an estimate of cardiac output, was calculated as the product of the integrated velocity curve and the area of the dorsal aorta (4). This method quantifies greater than $90 \%$ of the total stroke volume circulating in the system, inasmuch as only a small portion of the blood flows cephalad to the point of measurement (5). Passive filling volume equalled dorsal aortic stroke volume multiplied by the fraction of passive area. Active filling volume equalled dorsal aortic stroke volume multiplied by the fraction of the active area. Cycle length was defined as the time between the consecutive end-diastolic pressures.

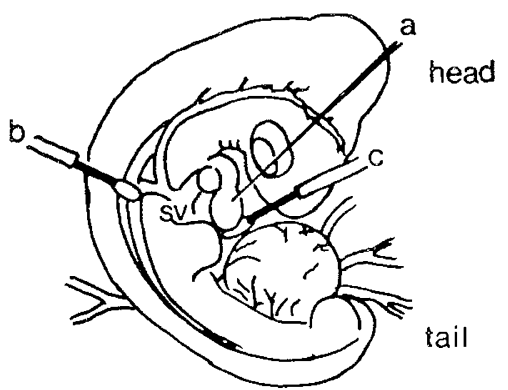

Fig. 1. An illustration of a stage 24 chick embryo with $(a)$ a glass cannula inserted into the ventricular cavity for measuring ventricular pressure and two piezoelectric crystals positioned $(b)$ over dorsal aorta for measuring dorsal aortic blood flow and $(c)$ at the apex of the ventricle aiming toward the atrioventricular orifice for measuring atrioventricular velocity ( $s v$, sinus venosus).
Six or more embryos were measured at each stage. The data are presented as mean \pm SEM. We did statistical comparison by analysis of variance, Tukey's honestly significant difference test $(8)$, and regression analysis. Significance level was defined as a $p$ value of less than $5 \%(p<0.05)$.

\section{RESULTS}

Cycle lengths across the stages were similar, ranging from 480 to $600 \mathrm{~ms}$ corresponding to heart rates between 100 to 125 beats/ min (Table 1). End-diastolic pressure doubled from stage 12 to stage 27 (Fig. 4). Over the same period, dorsal aortic stroke volume increased approximately 200 -fold (Fig. 5). Passive and active filling volume were $0.0038 \pm 0.0005$ and $0.0004 \pm 0.0002$ $\mathrm{mm}^{3}$, respectively, at stage 12 and increased to $0.23 \pm 0.09$ and $0.58 \pm 0.09 \mathrm{~mm}^{3}$, respectively, at stage 27 (Fig. 5). At stage 12, the passive filling volume accounted for $92 \%$ of the stroke volume. At stage 18, the passive and active filling volumes were similar. At stage 27 , the passive filling volume was $24 \%$ of the stroke volume. The ratio of passive to active filling volume decreased steeply from 7.89 at stage 12 to 1.21 at stage $18(\mathrm{y}=$ $21.57-1.14 \mathrm{x}, r=0.62$, standard error of the estimate $=0.29$ ), and then gradually to 0.35 at stage $27(\mathrm{y}=3.33-0.11 \mathrm{x}, r=$ 0.75 , standard error of the estimate $=0.02)($ Fig. 6$)$.

\section{DISCUSSION}

The relative proportion of passive and active diastolic filling changed as the ventricle developed from a smooth-walled cardiac tube to a trabecular sponge-like chamber. The heart begins as a muscle-wrapped tube formed by the fusion of the bilateral cardiac primordia in the lateral splanchnic folds. In the chick embryo, the heart begins to beat at stage $10(36 \mathrm{~h})$ and circulation is established shortly before stage $12(42 \mathrm{~h})(9)$. At stage 12 , the endocardial surface of the cardiac loop is smooth. At stage 18 (3 d), the trabecular spaces form in the ventricular wall, and the process of trabeculation continues as the heart grows. By stage 27 , the left and right ventricular chambers have a sponge-like network of trabecular struts (1). As the ventricular chambers expand, the muscular interventricular septum remains behind $(10,11)$. Finally, a dense ventricular wall forms when the trabeculae compact and some of the trabecular spaces are assimilated into the coronary vascular bed (12). The trabecular struts may act to limit wall motion. Thus, changes in the geometry and structure of the ventricular wall may affect the stiffness of the ventricular chambers.

The increase in ventricular end-diastolic pressure from stage

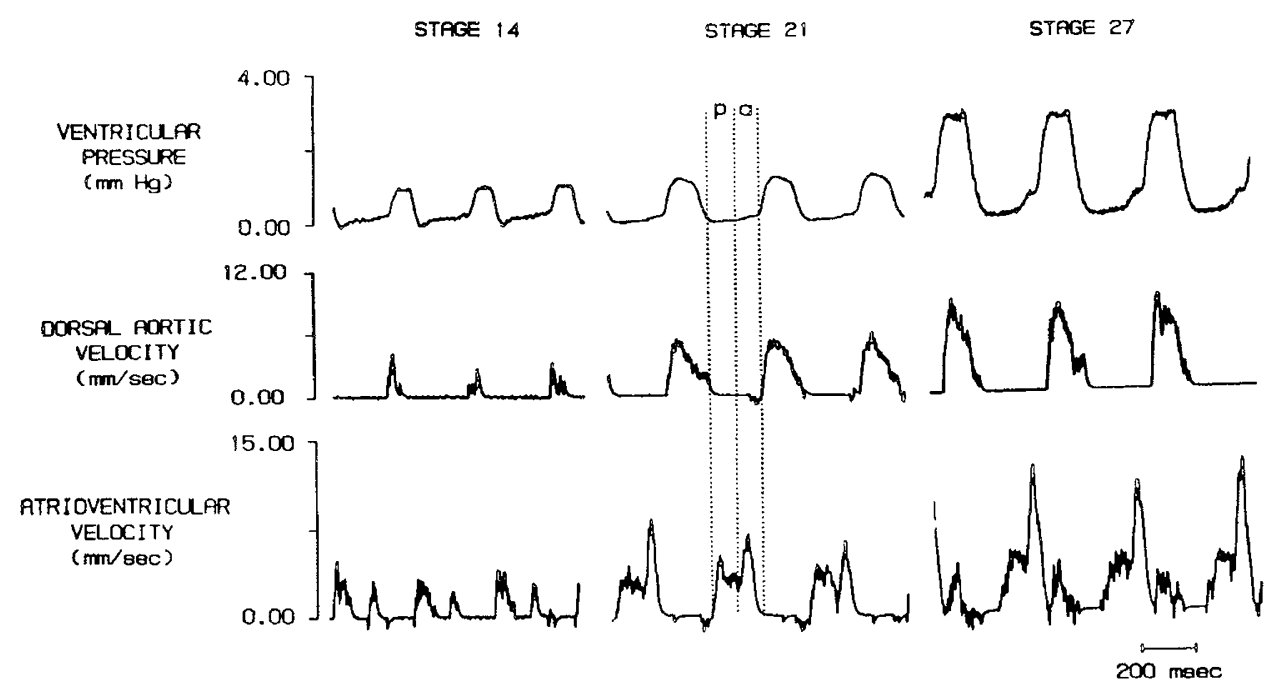

Fig. 2. An example of the simultaneously recorded tracings of ventricular pressure and dorsal aortic and atrioventricular velocities in a stage 21 chick embryo. Diastole is shown partitioned into two components: the passive phase indicated by $p$, and the active phase indicated by $a$. ( $m s e c$, ms). 


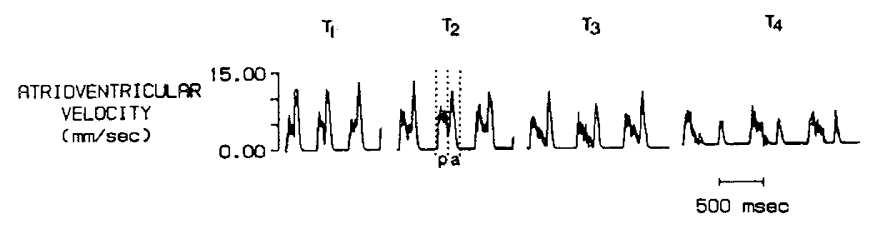

Fig. 3. Atrioventricular velocity tracing of stage 21 at different cycle lengths: $T_{1}=360 \mathrm{~ms}, T_{2}=430 \mathrm{~ms}, T_{3}=530 \mathrm{~ms}$, and $T_{4}=660 \mathrm{~ms}$. Although atrioventricular velocity is dependent on heart rates, passive $(p)$ component becomes progressively dominant as the cycle length increases ( $a$, active phase; $m s e c, \mathrm{~ms}$ ).

Table 1. Cycle length of stage 12 to 27 chick embryo

\begin{tabular}{crc}
\hline Stage & $n$ & Cycle length (ms) $^{*}$ \\
\hline 12 & 6 & $527 \pm 71$ \\
14 & 7 & $507 \pm 37$ \\
16 & 9 & $568 \pm 41$ \\
18 & 6 & $516 \pm 22$ \\
21 & 6 & $484 \pm 24$ \\
24 & 11 & $499 \pm 34$ \\
27 & 6 & $598 \pm 39$ \\
\hline
\end{tabular}

$*$ Mean \pm SEM

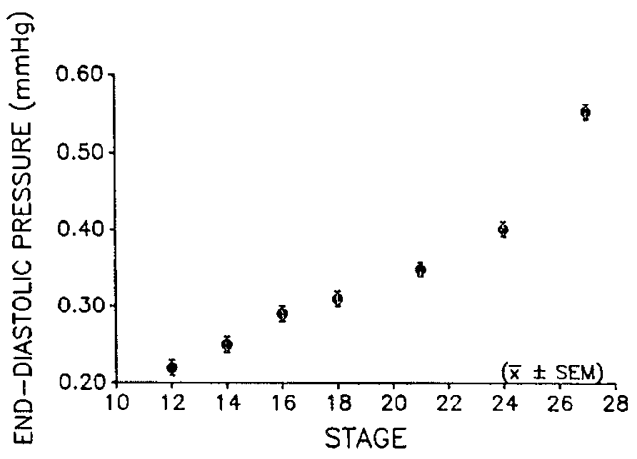

Fig. 4. Relationship of ventricular end-diastolic pressure with stage of development ( $n \geq 6$ at each stage).

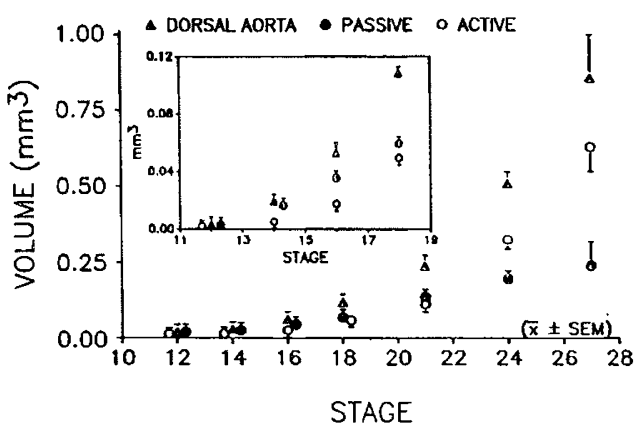

Fig. 5. Relationship of dorsal aortic stroke volume and atrioventricular filling volume with stage of development ( $n \geq 6$ at each stage). Dorsal aortic stroke volume $(\boldsymbol{\Lambda})$ increases with stages. Passive filling volume $(-)$ is dominant until stage 18 , then active filling volume $(O)$ progressively increases. Insert shows similar plot of volumes at stages 12 to 18 .

12 to 27 suggests that myocardial wall compliance decreased across this time. The shift from predominantly passive to active ventricular filling that occurred by stage 21 is consistent with a decrease in compliance after ventricular trabeculation. The accentuation in the end-diastole pressure may be due to the increase in force of atrial contraction, thus augmenting the active component of ventricular filling. Shortening fraction of ventricular cross-sectional area also increases from stage 12 to 18 , then decreases through stage 24 (13). The decrease in the shortening

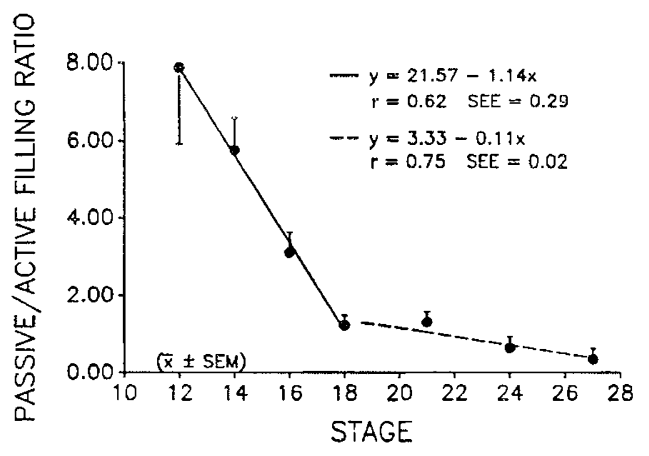

Fig. 6. Relationship of passive to active filling ratio with stage development ( $n \geq 6$ at each stage). There is a sharp decline of the passive/ active ratio at the early somite stages, and the gradient becomes less prominent after stage 18 .

fraction that occurs after stage 18 correlates with an increasing systolic ventricular stiffness and with the observed changes in diastolic filling.

In addition to the maturation of ventricular wall, differentiation also occurs within and around myocytes. Myofibrils increase in number and alignment, and ventricular size and weight increase $(14,15)$. The addition and organization of contractile elements within myocytes may limit cell distensibility. Interactions of cell with extracellular matrix and cell-to-cell adhesions may affect the mechanical characteristics of the ventricle. Before the trabeculation of the heart, the biomechanical force from the myocardial mantle is transmitted through cardiac jelly to the blood contained in the ventricular lumen (16). The myocytes are woven together by cell-to-cell adhesions. These adhesions include desmosomes and collagens within the matrix (17).

In the mature heart, calcium regulation during the cardiac cycle influences the diastolic properties of the mature myocardium (18). The availability of calcium is regulated by the complex interaction of multiple ion channels, intracellular ion stores, and the turnover of calcium-binding proteins. Relaxation can be partitioned into an early active phase due to active calcium redistribution and a later passive phase when calcium is extruded across the sarcolemma (19). The rate of relaxation then influences the stiffness of the individual sarcomere to deforming forces of the subsequent systole. Thus, biochemical events of diastolic relaxation may also affect the ventricular compliance.

Heart rate can profoundly affect the proportion of passive and active diastolic filling, as well as decrease the total integral of atrioventricular velocity. In development, heart rate increases with stage (5). With decreasing heart rate, there is a reciprocal relation between passive and active filling optimizing the stroke volume (20). Temperature affects pacemaker rate and cardiac output, whereas stroke volume remains constant, which is a protective cardiovascular mechanism (21). In the chick heart, pacing-induced increase in heart rate diminishes passive filling so that ventricular filling is primarily by active flow (22). For this reason, we chose to compare embryos at similar rates to permit analysis independent of rate.

Changes in diastolic properties may be an important epigenetic factor in cardiac morphogenesis. The embryonic heart adjusts ventricular mass to match work load (23). Alteration in intracardiac blood flow pattern may also affect the formation of the ventricular chambers. Partial occlusion of the left atrium by clipping or suture produces a spectrum of hypoplastic left ventricle $(24,25)$. The atrioventricular flow pattern may be a mechanical-biologic link to ventricular chamber growth and a determination of ventricular chamber volume.

Acknowledgment. The authors thank Jarle Holen, M.D., for his assistance in frequency analysis of the Doppler system. 


\section{REFERENCES}

1. Ben-Schachar G, Arcilla RA, Lucas RV, Manasek FJ 1985 Ventricular trabeculations in the chick embryo heart and their contribution to ventricular and muscular septal development. Circ Res 57:759-766

2. Reed KL, Sahn DJ, Scagnell S, Anderson CF, Shenker L 1986 Doppler echocardiographic studies of diastolic function in human fetal heart: changes during gestation. J Am Coll Cardiol 9:391-395

3. Hamburger V, Hamilton HL 1951 A series of normal stages in the development of the chick embryo. J Morphol 88:49-92

4. Clark EB, Hu N 1982 Developmental hemodynamic changes in the chick cmbryo stages 18 to 27 . Circ Res 51:810-815

5. Hu N, Clark EB 1989 Hemodynamics of the stage 12 to 29 chick embryo. Circ Res 65:1665-1670

6. Hay DA, Low FN 1970 The fusion of dorsal and ventral endocardial cushions in the embryonic chick heart: a study in fine structure. Am J Anat 133:124

7. McDonald DA 1974 Blood Flow in Arteries. Edward Arnold Publishers Ltd London, pp 55-70

8. Tukey JW 1949 Comparing individual means in the analysis of variance. Biometrics 5:99-114

9. Clark EB 1989 Growth, morphogenesis, and function: the dynamics of cardiac development. In: Moller JH, Neal W, Lock J (eds) Fetal, Neonatal and Infant Heart Disease. Appleton-Century-Crofts, New York, pp 1-17

10. Harh JY, Paul MH 1975 Experimental cardiac morphogenesis. I. Development of the ventricular septum in the chick. J Embryol Exp Morphol 33:13-28

11. Van Mierop LHS 1962 Morphological development of the heart. In: Berne RN. Sperelakis N. Geiger SR (eds) Handbook of Physiology. The Cardiovascular System. Williams and Wilkins, Baltimore, pp 1-28

12. Rychterova V 1971 Principle of growth in the thickness of the heart ventricular wall in the chick embryo. Folia Morphol (Praha) 19:262-272

13. Keller BB, Hu N. Clark EB 1990 Correlation of ventricular area, perimeter and conotruncal diameter with ventricular mass and function in the stage 12 to 24 chick embryo. Circ Res 66:109-114
14. Clark EB, Hu N, Vandekieft GK. Olson C. Tomanek RJ 1986 Ventricular function and morphology in the chick embryo from stages 18 to 29 . Am J Physiol 250:H407-H413

15. Manasek FJ 1970 Histogenesis of the embryonic myocardium. Am J Cardiol 25:149-168

16. Nakamura A, Manasek FJ 1981 An experimental study of the relation of cardiac jelly to the shape of the early chick embryonic heart. J Embryol Exp Morphol 65:235-256

17. Borg TK Raso DS. Terracio L 1990 Potential role of the extracellular matrix in post-septation development of the heart. In: Bockman DE, Kirby ML (eds) Embryonic Origins of Defective Heart Development, Vol. 588. Annals of the New York Academy of Sciences, New York, pp 87-92

18. Morgan JP, MacKinnon R, Briggs M. Gwathmey JK 1988 Calcium and cardiac relaxation. In: Grossman W, Lorell BH (eds) Diastolic Relaxation of the Heart. Martinus Nijhoff, Boston, pp 17-26

19. Barry WH 1988 Cellular mechanisms of relaxation: lessons from frogs, birds, and mammals. In: Grossman W, Lorell BH (eds) Diastolic Relaxation of the Heart. Martinus Nijhoff, Boston, pp 3-10

20. Hu N, Keller BB, Clark EB 1989 Relationship of cycle length, diastolic filling and ventricular performance in the stage 21 chick. Pediatr Res 25:25A (abstr)

21. Wispe J, Hu N, Clark EB 1983 Effect of environmental hypothermia on dorsal aortic blood flow in the chick embryo, stages 18 to 24 . Pediatr Res 17:945948

22. Dunnigan A, Hu N, Benson Jr DW, Clark EB 1987 Effect of heart rate increase on dorsal aortic flow in the stage 24 chick embryo. Pediatr Res 22:442-444

23. Clark EB, Hu N, Frommelt P, Vandekieft GK, Dummett JL. Tomanek RJ 1989 Effect of increased pressure on ventricular growth in stage 21 chick embryo. Am J Physiol 257:H55-H66

24. Rychter Z, Lemez L 1962 The suppression of the left atrial anlage relating to the origin of the coarctation of aorta in chick embryos. In: Symposium Proceedings, Experimental Embryology and Neuroanatomy. Publishing House of the Czechoslovak Academy of Sciences, Prague, pp 35-43

25. Sweeny LJ 1981 Morphometric Analysis of an Experimental Model of Left Heart Hypoplasia in the Chick. University of Nebraska, Omaha, 137 pp 